\title{
Right ventricular dysfunction in children supported with pulsatile ventricular assist devices
}

\author{
Ann Karimova, MD, ${ }^{a}$ Charissa R. Pockett, MD, ${ }^{\mathrm{b}}$ Nagore Lasuen, MD, ${ }^{\mathrm{a}}$ Nathalie Dedieu, MD, ${ }^{\mathrm{a}}$ \\ Jennifer Rutledge, MD, ${ }^{b}$ Mathew Fenton, MD, ${ }^{a}$ Christina VanderPluym, MD, ${ }^{c}$ Ivan M. Rebeyka, MD, \\ Troy E. Dominguez, MD, ${ }^{a}$ and Holger Buchholz, $\mathrm{MD}^{\mathrm{b}}$
}

Objectives: To describe the incidence and severity of right ventricular dysfunction (RVD) in pediatric ventric-
ular assist device (VAD) recipients and to identify the preoperative characteristics associated with RVD and their effect on outcomes. Methods: Children bridged to transplantation from 2004 to 2011 were included. RVD was defined as the use of a
left VAD (LVAD) with an elevated central venous pressure of $>16 \mathrm{~mm} \mathrm{Hg}$ with inotropic therapy and/or inhaled
nitric oxide for $>96$ hours or biventricular assist (BiVAD).

Results: A total of 57 children (median age, 2.97 years; range 35 days to 15.8 years) were supported. Of the 57, $43(75 \%)$ had an LVAD, and of those, 10 developed RVD. The remaining $14(25 \%)$ required BiVAD. Thus, RVD occurred in 24 of 57 patients $(42 \%)$. Preoperative variables such as younger age $(P=.01)$, use of extracorporeal mechanical support $(P=.006)$, and elevated urea $(P=.03)$, creatinine $(P=.02)$, and bilirubin $(P=.001)$ were associated with RVD. Multiple logistic regression analysis indicated that elevated urea and extracorporeal mechanical support (odds ratio, 26.4; 95\% confidence interval, 2.3-307.3; and odds ratio, 27.8; 95\% confidence interval, 2.5-312.3, respectively) were risk factors for BiVAD. The patients who developed RVD on LVAD had a complicated postoperative course but excellent survival (100\%), comparable to those with preserved right ventricular function $(91 \%)$. The survival for those requiring BiVAD was reduced $(71 \%)$.

Conclusions: RVD occurred in approximately $40 \%$ of pediatric VAD recipients and affects their peri-implantation morbidity and bridging outcomes. Preoperative extracorporeal membrane oxygenation and elevated urea were risk factors for BiVAD. Additional studies of the management of RVD in children after VAD implantation are warranted. (J Thorac Cardiovasc Surg 2014;147:1691-7)

Supplemental material is available online.

Mechanical bridging with ventricular assist devices (VADs) has recently become an effective treatment option for children awaiting heart transplantation. The Berlin Heart EXCOR Pediatric VAD (Berlin, Germany) has been used worldwide, and substantial experience is now available, with the indications, complications, and outcomes of EXCOR use in children. ${ }^{1-3}$

\footnotetext{
From the Cardiothoracic Unit, ${ }^{\mathrm{a}}$ Great Ormond Street Hospital for Children, London, United Kingdom; Department of Pediatric Cardiology, ${ }^{b}$ Stollery Children's Hospital, Edmonton, Alberta, Canada; and Department of Cardiology, ${ }^{\mathrm{c}}$ Boston Children's Hospital, Boston, Mass.

Disclosures: Authors have nothing to disclose with regard to commercial support.

Drs Dominguez and Buchholz were co-senior authors.

Received for publication May 15, 2013; revisions received Oct 29, 2013; accepted for publication Nov 8, 2013; available ahead of print Dec 16, 2013.

Address for reprints: Ann Karimova, MD, Cardiothoracic Unit, Great Ormond Street Hospital for Children, Great Ormond St, London, WC1N 3JH UK (E-mail: Ann.Karimova@gosh.nhs.uk).

0022-5223/ $\$ 36.00$

Copyright (c) 2014 by The American Association for Thoracic Surgery

http://dx.doi.org/10.1016/j.jtcvs.2013.11.012
}

An important question that has not been addressed in children is the right ventricular (RV) response to implantation of a left ventricular assist device (LVAD) and how to distinguish those patients who will require biventricular assist device (BiVAD).

In adults, considerable research has been done on RV dysfunction (RVD) after LVAD implantation showing that RVD is 1 of the key determinants of the bridging outcome. Preoperative risk factors have been identified and used to assess the likelihood of RV compromise in adult VAD recipients. $^{4-8}$

However, some of the identified risk factors for RVD in adults might not exist or be applicable to children. Moreover, several reports of VAD use in children have highlighted a reduction in the use of BiVAD over time; however, the criteria for mechanical RV support in children have varied among centers. ${ }^{1,3}$

The present study aimed to describe the incidence and severity of RVD in children bridged with the Berlin Heart EXCOR VAD; to identify the preoperative characteristics associated with RVD requiring a BiVAD; and to assess the effect RVD has on postoperative morbidity and outcomes. 


$$
\begin{aligned}
& \text { Abbreviations and Acronyms } \\
& \text { BiVAD = biventricular assist device } \\
& \text { DCM = dilated cardiomyopathy } \\
& \text { ECMO }=\text { extracorporeal membrane oxygenation } \\
& \text { LVAD }=\text { left ventricular assist device } \\
& \mathrm{OR} \quad=\text { odds ratio } \\
& \text { RRT = renal replacement therapy } \\
& \mathrm{RV}=\text { right ventricular } \\
& \text { RVAD = right ventricular assist device } \\
& \text { RVD = right ventricular dysfunction } \\
& \mathrm{VAD}=\text { ventricular assist device }
\end{aligned}
$$

\section{METHODS}

The present study was a retrospective, observational cohort study using the data from patients treated in 2 tertiary-care pediatric transplant centers from November 2004 to January 2011. All patients aged $<16$ years who were bridged to transplantation with the Berlin Heart EXCOR Pediatric VAD were identified at these centers from the databases. The databases included prospective information on the patient demographic data, VAD implantation details, and outcomes. Additional data for the study were gathered retrospectively (see the section "Data Collection"). The respective institutional research ethics review boards of the 2 centers approved the study, and a waiver of informed consent was obtained.

\section{VAD Management}

All children were supported using a Berlin Heart EXCOR Pediatric VAD. The 2 study sites had a uniform approach to VAD implantation, with the expectation of LVAD only support in most cases. The pump size was chosen according to the Berlin Heart Company protocol, and the VAD rate was set to achieve a cardiac index of 2.4 to $2.8 \mathrm{~L} / \mathrm{min} / \mathrm{m}^{2}$. Left ventricular unloading, cannula placement, and RV function were assessed using intraoperative transesophageal echocardiography. When weaning off cardiopulmonary bypass, the circulating blood volume was optimized and the right ventricle was supported with inotropes, inhaled nitric oxide, and pacing, if necessary. The addition of a right VAD (RVAD) was reserved for patients who could not separate from cardiopulmonary bypass despite optimal medical right heart support. The strategy for treating children with a VAD, including anticoagulation, has been previously published. ${ }^{10}$

\section{Data Collection}

Preoperative and operative data. The preimplantation data included the patient demographic data, diagnosis, mechanical ventilation and intravenous inotropic support duration, extracorporeal membrane oxygenation (ECMO) period, highest central venous pressure within 24 hours before VAD implantation, and laboratory test results (hepatic and renal function, blood count, and coagulation status). The preimplantation transthoracic echocardiograms were also reviewed by a cardiologist who was unaware of the treatment group with attention to the following: right atrial and left atrial area, tricuspid regurgitation grade ( 0 , none; 1 , mild; 2 , moderate; and 3, severe), tricuspid regurgitation velocity (an estimate of RV systolic pressure), tricuspid valve annulus size, RV end-diastolic dimension, and qualitative RV function ( 0 , normal; 1 , mild dysfunction; 2, moderate dysfunction; and 3, severe dysfunction). The operative data included the duration of cardiopulmonary bypass and transfusion of red blood cells and other blood products within the first 24 hours after implantation.

\section{Outcomes}

RV dysfunction. RVD was defined as LVAD with an elevated central venous pressure $>16 \mathrm{~mm} \mathrm{Hg}$ with inotropes (other than milrinone) and/or inhaled nitric oxide therapy $>96$ hours, or a need for BiVAD. Other conditions with similar clinical signs were excluded (eg, tamponade, pneumothorax, effusions, or ventricular arrhythmia).

According to the increasing severity of RVD, the patients were divided into the following categories: LVAD with no RVD (LVAD-only group), LVAD with medically managed RVD (LVAD-RVD group); and LVAD with RVD requiring an RVAD (BiVAD group).

Morbidity and mortality. Postoperative complications, such as bleeding, a requirement for mediastinal exploration or placement of a chest drain, and renal failure requiring renal replacement therapy (RRT) with peritoneal dialysis or hemofiltration, were recorded. The duration of postoperative mechanical ventilation after VAD implantation was recorded. In-hospital mortality was also recorded.

\section{Statistical Analysis}

Preoperative and operative variables and RVD. Continuous data are presented as the mean \pm standard deviation for normally distributed data or the median and range otherwise. The categorical and ordinal data are presented as numbers and percentages. Comparisons between groups using continuous data were performed using analysis of variance for normally distributed data and the Kruskal-Wallis test otherwise. For comparisons using categorical or ordinal data, the $\chi^{2}$ or Fisher exact test was performed. Assuming the 3 groups (LVAD-only, LVAD-RVD, and BiVAD) represented an ordered group (increasing levels of RVD), a nonparametric (Wilcoxon-type) test for trend was performed to assess the trends in continuous variables across the 3 ordered groups.

Risk factors for BiVAD. For the assessment of the risk factors for BiVAD, all patients treated with LVAD only were used as the reference group. Comparisons using continuous variables were performed using the Student's $t$ test for normally distributed data or the Wilcoxon rank sum test otherwise. Comparisons using ordinal or categorical data were performed using the $\chi^{2}$ or Fisher exact test. Variables identified as significantly associated with the need for BiVAD support were entered into a multiple logistic regression model to identify the independent risk factors for BiVAD implantation and calculation of the odds ratios (ORs) and their respective $95 \%$ confidence intervals.

Outcomes after RVD. Comparisons of the outcomes were performed for all 3 groups. Additionally, we sought to determine whether any difference was present in the outcomes in the LVAD-RVD group versus the LVAD group without RVD. Differences in the duration of postoperative ventilation and hospital length of stay were analyzed using a KruskalWallis test or Wilcoxon rank sum test. Differences in the complication rates and in-hospital mortality were assessed using the $\chi^{2}$ test or Fisher exact test.

Statistical analysis was performed using Stata, version 9.2 (StataCorp LP, College Station, Tex).

\section{RESULTS \\ Baseline Demographics}

A total of 57 children ( 28 males and 29 females) at a median age of 2.97 years (range, 35 days to 15.8 years) and median weight of $12.8 \mathrm{~kg}$ (range, 3.6-90) underwent VAD implantation during the study period. In all patients, the indication for VAD support was end-stage heart failure with the intention to bridge the patient to cardiac transplantation. Five patients with congenital heart disease (all with single ventricle physiology) who were bridged during the study period were excluded from the present study. 
Patients with congenital heart disease and biventricular circulation had not undergone VAD implantation during the study period. Of the 57 included children, $43(75 \%)$ were supported with LVAD and $14(25 \%)$ with BiVAD. Most of the children, $42(74 \%)$, had a diagnosis of dilated cardiomyopathy (DCM). The remaining 15 children $(26 \%)$ were grouped together as a non-DCM group and had a diagnosis of confirmed viral myocarditis in 5, left ventricular noncompaction in 3 , restrictive cardiomyopathy in 2, myocardial dysfunction secondary to complications of Kawasaki disease in 2, rheumatic heart disease in 2, and hypertrophic cardiomyopathy in 1 .

\section{RVD Incidence}

Of the 43 patients supported with an LVAD, 33 (77\%) had preserved right heart function (LVAD-only), and 10 (23\%) had developed RVD (LVAD-RVD). Inhaled nitric oxide was used in 6 of the 10 LVAD-RVD patients $(60 \%)$. Of the 14 patients requiring BiVAD, the RVAD was implanted at LVAD implantation in all but 1 patient, who required RVAD implantation on day 22 of support for persistent RVD complicated by sepsis (Figure 1).

\section{RVD and Demographic and Perioperative Data}

The associations between the severity of RVD and the demographic and preoperative data are listed in Table 1. Differences in the underlying cardiac diagnosis were of borderline statistical significance among the groups, with the DCM diagnosis occurring more frequently in the LVAD-only group. Increased RVD severity was also associated with increasing patient weight and age and derangement of preoperative renal and hepatic function. ECMO had frequently been used before VAD implantation ( 24 of 57 [42.1\%]), and the patients in the BiVAD group had received this therapy most frequently.

The only variable identified from the preoperative echocardiographic findings that was associated with postoperative RVD was a qualitative impairment in RV function (LVAD-only, median, 0 [range, 0-2]; vs LVADRVD, median, 0.5 [range, 0-3]; vs BiVAD, median 2 [range, 0-3]; $P=.01$ ). None of the other preoperative echocardiographic variables were significantly different across the 3 groups of patients (Table E1).

The intraoperative variables for the 3 groups are listed in Table 2. In the patients successfully treated with an LVAD, neither the cardiopulmonary bypass duration $(P=.7)$ nor the transfusion volumes $(P=.17)$ were different between the patients with and without RVD.

\section{Risk Factors for BiVAD}

BiVAD was used in 14 of the 57 patients $(24.6 \%)$, and the rate of BiVAD use did not differ by year of implantation $(P=.59)$. Compared with the LVAD patients, the BiVAD patients were significantly older (median age, 7.5 years;

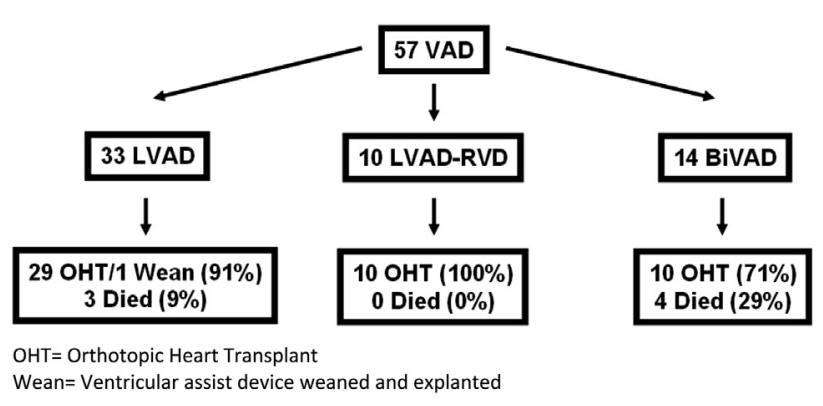

FIGURE 1. Proportions and survival rates of patients with a left ventricular assist device ( $L V A D)$ with and without right ventricular dysfunction $(R V D)$ and those with a biventricular assist device (BiVAD). VAD, Ventricular assist device; OHT, orthotopic heart transplant; Wean, ventricular assist device weaned and explanted.

range, 3.7 months to 15.8 years; vs 1.8 years, range, 1.2 months to 15.7 years; $P=.02$ ). Only 1 of the 16 patients aged $<1$ year required a BiVAD. The BiVAD patients were also more likely to have received ECMO before VAD implantation (11 of 14 [79\%] vs 13 of 43 [30\%]; $P=.02)$. Compared with the patients with DCM, a trend was seen for the non-DCM group to require BiVAD more frequently ( 8 of $42[19 \%]$ vs 6 of $15[40 \%], P=.1$ ). The preoperative laboratory study results demonstrated significantly greater levels of urea (median, $10.2 \mathrm{mmol} / \mathrm{L}$; range, 3.6-36.1; vs 6 , range, $1.5-29.0 \mathrm{mmol} / \mathrm{L}, P=.03$ ), alanine aminotransferase (median, $84.5 \mu \mathrm{kat} / \mathrm{L}$, range, 23-1780; vs $39 \mu \mathrm{kat} / \mathrm{L}$; range, 6-1610; $P=.034$ ), and bilirubin (median, $31.5 \mu \mathrm{mol} / \mathrm{L}$; range, 6-246; vs 11.5, range, 2-109; $P=.02$ ) in patients requiring BiVAD compared with those requiring LVAD.

Multiple logistic regression analysis was performed to identify the independent risk factors for BiVAD implantation, including age, pre-implantation urea, and use of ECMO in the model. Serum urea was entered as a dichotomous variable, defined by an abnormal value of $>10 \mathrm{mmol} / \mathrm{L}$. Hepatic function tests were not included in the analysis because test results were not available for $25 \%$ of the patients. Only the urea level and ECMO use before implantation were statistically significant (OR, 26.4; 95\% CI, 2.3-307.3; and OR, 27.8, 95\% CI, 2.5-312.3, respectively). A trend in significance was found for infants (aged $<1$ year) to require BiVAD less frequently (OR, 0.09; 95\% CI, 0.01-1.2).

\section{Postoperative Morbidity and Survival}

The postimplantation morbidity of the 3 groups is listed in Table 3. RRT was required in up to one third of the BiVAD and LVAD-RVD patients, whereas the need for RRT in the LVAD-only group was infrequent (4 of 12 [33\%] vs 4 of $10[40 \%]$ vs 2 of $33[6 \%] ; P=.02$ ). In all surviving patients, renal function recovered sufficiently for the patients to be weaned off RRT before heart transplantation. 
TABLE 1. Demographic and pre-VAD implantation characteristics

\begin{tabular}{|c|c|c|c|c|}
\hline Characteristic & LVAD only $(\mathrm{n}=33)$ & LVAD-RVD $(n=10)$ & $\operatorname{BiVAD}(n=14)$ & $P$ value \\
\hline DCM & $28(85)$ & $6(60)$ & $8(57)$ & .08 \\
\hline Female gender & $18(55)$ & $6(60)$ & $5(36)$ & .45 \\
\hline Weight (kg) & $11(3.8-68)$ & $17.5(3.6-80)$ & $19(6.2-90)$ & .04 \\
\hline Age (y) & $1.7(36 \mathrm{~d}$ to $15.7 \mathrm{y})$ & $3.9(35 \mathrm{~d}$ to $15.4 \mathrm{y})$ & $8.9(110 \mathrm{~d}$ to $15.8 \mathrm{y})$ & .012 \\
\hline CVP (mm Hg) & $10(5-25)$ & $14.5(5-19)$ & $16.5(13-21)$ & .004 \\
\hline Inotropes (d) & $10(1-52)$ & $14(5-132)$ & $6(0-51)$ & .44 \\
\hline \multicolumn{5}{|l|}{ Inotropes } \\
\hline Milrinone & $30(91)$ & $10(100)$ & $10(71)$ & .11 \\
\hline Adrenaline & $17(51.5)$ & $5(50)$ & $9(64.3)$ & .69 \\
\hline Noradrenaline & $2(6.1)$ & $1(10)$ & $4(28.6)$ & .09 \\
\hline Dopamine & $6(18.2)$ & $2(20)$ & $1(7.2)$ & .68 \\
\hline Dobutamine & $14(42.4)$ & $4(40)$ & $4(28.6)$ & .7 \\
\hline Antiarrhythmics & $4(16.1)$ & $1(10)$ & $4(28.6)$ & .15 \\
\hline Mechanical ventilation & $25(75.8)$ & $5(50)$ & $12(85.7)$ & .15 \\
\hline Ventilation (d) & $9.5(0-44)$ & $2(0-45)$ & $7(0-49)$ & .53 \\
\hline RRT & $1(3)$ & $0(0)$ & $3(21.8)$ & .07 \\
\hline ECMO & $10(30)$ & $3(30)$ & $11(79)$ & .006 \\
\hline ECMO duration (d) & $0(0-38)$ & $0(0-40)$ & $5.5(0-30)$ & .077 \\
\hline Urea $(\mathrm{mmo} / \mathrm{L})$ & $6(1.5-20.3)$ & $7.6(3.5-29)$ & $10.2(3.6-36.1)$ & .026 \\
\hline Creatinine $(\mu \mathrm{mol} / \mathrm{L})$ & 35 (4.2-89) & $73(25-86)$ & $55.5(19-192)$ & .026 \\
\hline $\mathrm{PT}(\mathrm{s})$ & $13.5(10.3-163)$ & $15.2(10-100)$ & $21.4(10.8-96)$ & .13 \\
\hline Bilirubin $(\mu \mathrm{mol} / \mathrm{L})^{*}$ & $10(2-100)$ & $26(11-109)$ & $31.5(6-246)$ & .001 \\
\hline $\operatorname{ALT}(\mu \mathrm{kat} / \mathrm{L}) \dagger$ & $29(6-1610)$ & $93(17-1260)$ & $84.5(23-1780)$ & .005 \\
\hline Sodium $(\mathrm{mmol} / \mathrm{L})$ & $138(123-163)$ & $135.5(120-148)$ & $143.5(132-156)$ & .2 \\
\hline
\end{tabular}

The overall survival to hospital discharge was $88 \%$ (50 of 57). Of the survivors, 49 patients survived to transplantation and 1 underwent VAD explantation and was discharged home. The survival was similar in the children with and without RVD treated with LVAD support (Figure 1); however, it was lower in the BiVAD group (OR, 0.19; 95\% CI, 0.03-0.98). The details of the nonsurvivors are listed in Table 4. Of the 7 patients who died, 2 died intraoperatively (both patients were treated during the first year of the VAD program and they both died of multiorgan failure after late conversion from ECMO).

\section{DISCUSSION}

The results of the present study have demonstrated that there is a spectrum of response in RV performance to VAD implantation in children. Most children can be bridged to transplantation with an LVAD only, although approximately 1 in 4 LVAD recipients developed RVD and needed aggressive pharmacologic RV support and a longer postoperative course in intensive care. Their overall survival, however, was excellent. Severe RVD requiring BiVAD support occurred in $25 \%$ of the VAD recipients, consistent with the rate of previous studies, and those patients had significantly reduced survival. ${ }^{1,3,9}$

These findings suggest that a period of medical management for patients with RVD that develops after LVAD implantation (which can persist for several days) is warranted in most cases.

\section{Right Ventricle and VAD}

Deterioration of RV function is an acute event observed in a significant proportion of patients after LVAD implantation. It has been studied in adults and recognized as one of the determinants of morbidity and mortality. ${ }^{4,11-13}$ Several

TABLE 2. Intraoperative characteristics

\begin{tabular}{|c|c|c|c|c|}
\hline Characteristic & LVAD only $(n=33)$ & LVAD-RVD $(\mathbf{n}=10)$ & $\operatorname{BiVAD}(n=14)$ & $P$ value \\
\hline $\mathrm{CPB}(\min )$ & $104(72-173)$ & $121(80-170)$ & $196(87-465)$ & .01 \\
\hline $\mathrm{RBC}(\mathrm{mL} / \mathrm{kg})$ & $46.8(9.7-169.0)$ & $18.3(6.5-109.1)$ & $37.9(16.0-201.6)$ & .15 \\
\hline Total blood products $(\mathrm{mL} / \mathrm{kg})$ & $72.9(16.0-241.0)$ & $54.4(6.5-142.0)$ & $75.0(37.3-233.9)$ & .31 \\
\hline Total blood products (mL) & $877.5(250-2100)$ & $730(176-1850)$ & $1900(370-7000)$ & .02 \\
\hline
\end{tabular}

Data presented as median (range). $L V A D$, Left ventricular assist device; $R V D$, right ventricular dysfunction; BiVAD, biventricular assist device; $C P B$, cardiopulmonary bypass; $R B C$, red blood cell. 
TABLE 3. Post-VAD implantation morbidity

\begin{tabular}{lccc}
\hline \multicolumn{1}{c}{ Variable } & LVAD only $(\mathbf{n}=\mathbf{3 3})$ & LVAD-RVD $(\mathbf{n}=\mathbf{1 0})$ & BiVAD $(\mathbf{n}=\mathbf{1 2}) *$ \\
\hline Postoperative bleeding $\dagger$ & $5 / 33(15)$ & $2 / 10(20)$ & $5 / 12(42)$ \\
Ventilation (d) & $5(1-24)$ & $9.5(3-22)$ & $6.5(2-176)$ \\
RRT & $2 / 33(6)$ & $4 / 10(40)$ & $4 / 12(33)$ \\
\hline$V A D$, value
\end{tabular}

$V A D$, Ventricular assist device; $L V A D$, left VAD; $R V D$, right ventricular dysfunction; BiVAD, biventricular assist device; RRT, renal replacement therapy. $*$ Two BiVAD patients died during implantation and were not included. †Requiring re-exploration or drain insertion.

mechanisms have been postulated to play a role in the development of RVD, including the effects of cardiopulmonary bypass and acute changes in the RV loading conditions and geometry. ${ }^{14}$ Recent studies in adults have aimed to identify the predictors of RVD preoperatively, and variables such as nonischemic etiology of heart failure, female gender, and elevated bilirubin have been identified. ${ }^{5,6,15}$ Compared with adults, our understanding of RV function after VAD implantation in pediatric patients is limited; however, preliminary data using the Interagency Registry for Mechanically Assisted Circulatory Support adverse events have confirmed the association of RVD with adverse outcomes in children. ${ }^{16}$

\section{Preimplantation Status and RVD}

Most children will have some degree of RV failure with organ congestion before VAD implantation. Our results have suggested that an important association might exist between preoperative RV impairment and organ congestion (as evidenced by the greater preoperative central venous pressure and deranged hepatic and renal function) and the development of RVD after VAD placement. These results could be helpful in identifying children at risk of perioperative RVD who would be likely to need either medical or mechanical RV support. This also underscores the importance of the timing of VAD implantation and optimization of the circulation before VAD implantation to reduce organ congestion.

\section{Preimplantation Diagnosis and RVD}

The preponderance of RVD in patients with a diagnosis other than DCM was a notable finding. One could speculate that in this heterogeneous group of diagnoses, the reasons for worse RV function could have been related to factors such as higher pulmonary vascular resistance in patients with restrictive physiology or primary right ventricular myocardial disease, such as in myocarditis. Perhaps more clinically relevant was the finding that most patients with DCM were able to be supported with an LVAD only and that unless exceptional peri-implantation complications occurred, LVAD is a suitable support choice. ${ }^{1}$

\section{Role of Echocardiography}

We found a limited role for preoperative echocardiography in the prediction of postimplantation RVD. The only preoperative echocardiographic variable associated with RVD was the qualitative assessment of RV function, a parameter known to be operator dependent and therefore of limited reproducibility. A similar result was previously reported analyzing the preoperative echocardiographic data from adult VAD recipients. ${ }^{12}$ Any comparison with adult data should be done with caution, because adult studies have included patients with continuous flow pumps and the etiology of heart failure is different from that of children. The limited value of preoperative echocardiography in predicting RVD in children in the present study was disappointing; however, more advanced echocardiographic techniques might be more helpful and require additional investigation. ${ }^{17,18}$

\section{RVD on LVAD}

Our definition of RVD was chosen to describe the spectrum of RVD in pediatric LVAD recipients and to distinguish between transient, medically manageable RVD

TABLE 4. Nonsurvivor patient characteristics (ordered by year of VAD implantation)

\begin{tabular}{lccllcc}
\hline VAD year & Age $(\mathbf{y})$ & Weight $(\mathbf{k g})$ & \multicolumn{1}{c}{ Diagnosis } & \multicolumn{1}{c}{ Support } & Support duration (d) & Cause of death \\
\hline 2006 & 15.8 & 90 & DCM & BiVAD & Died in OR* & $\begin{array}{c}\text { Sepsis, liver failure, bleeding } \\
\text { (conversion from ECMO) }\end{array}$ \\
2006 & 1.3 & 6.2 & DCM & BiVAD & Died in OR* & $\begin{array}{c}\text { Sepsis, multiorgan failure } \\
\text { (conversion from ECMO) }\end{array}$ \\
& & & & & 14 & Stroke \\
2007 & 4.1 & 16 & DCM & LVAD only & Sepsis, multiorgan failure \\
2007 & 11.5 & 46 & DCM & LVAD only & 14 & Sepsis, stroke \\
2008 & 0.3 & 4.3 & DCM & BiVAD (RVAD added day 22) & 25 & Sepsis, multiorgan failure \\
2010 & 6.0 & 15 & Non-DCM (restrictive) & BiVAD & 38 & Stroke \\
\hline
\end{tabular}

$V A D$, Ventricular assist device; DCM, dilated cardiomyopathy; BiVAD, biventricular assist device; $O R$, operating room; $E C M O$, extracorporeal membrane oxygenation; $L V A D$, left VAD; RVAD, right VAD. *Two patients (both implanted at the beginning of the VAD program) died during implantation; both had undergone late conversion from ECMO (after 15 and 30 days, respectively) with multiorgan failure and sepsis. 
and severe, refractory RVD necessitating mechanical support with an RVAD. Describing the patients receiving LVAD support who developed RVD is clinically important, because these will be the marginal patients for whom surgeons must make the difficult decision between persisting with LVAD at the cost of increased peri-implantation morbidity or proceeding to BiVAD. RVD can compromise both the LVAD and end organ function, and these patients will require longer inotropic support, prolonged ventilation, and, often, the use of RRT. ${ }^{19}$ However, with time, the RV function in these patients improved, their end organ function normalized, and their survival rate was excellent and comparable to that of those with an LVAD without RVD. To this end, our data showing RV recovery and good survival in the LVAD patients are informative and will encourage the use of LVAD in children.

Optimization of RV function starts preoperatively with aggressive diuresis, inotropes, and pulmonary vasodilators. ${ }^{20,21}$ In the peri-implantation phase, a "normal," rather than an excessive, LVAD output will ensure decompression of the left ventricle and prevent an acute increase in RV preload. Echocardiography can be used to assess the ventricular size, aiming to avoid an acute shift of the interventricular septum from right to left and worsening of tricuspid insufficiency. Finally, meticulous hemostasis, a low threshold for inotrope and pulmonary vasodilator use, and rhythm control are all important elements of RV support. ${ }^{9,22}$

\section{RVD Requiring BiVAD}

Great variability exists in practice in terms of BiVAD use among pediatric centers. One of the early reports of the use of the Berlin Heart EXCOR in children in North America reported elective use of BiVAD in all patients, with excellent survival. ${ }^{23}$ However, most centers are now much more restrictive in the use of BiVAD support, with BiVAD reserved only for cases with severe RVD that cannot be controlled medically. ${ }^{1,3,9,10,24}$

A systematic approach to decision making between LVAD and BiVAD for children has been recently reported using an algorithm using the peri-implantation clinical and echocardiographic data. ${ }^{10}$ This algorithm recognizes an intermediate category "between LVAD and BiVAD," giving the surgeon some flexibility to choose the type of support according to the intraoperative RV performance. Using this approach, the observed BiVAD rate was $35 \%$, slightly greater than that in our study. ${ }^{1,10}$

\section{Risk Factors for BiVAD}

ECMO is often used before VAD to either acutely stabilize patients in cardiogenic shock or as a bridge to decision. ${ }^{1}$ In our study, multivariate analysis identified $\mathrm{ECMO}$ as 1 of the independent risk factors for BiVAD. A similar finding was reported in another pediatric series. ${ }^{2}$
The explanation for this finding is likely multifactorial, including inadequate decompression of the left heart with ECMO, resulting in lung injury and elevated pulmonary vascular resistance, and ECMO-related complications such as infection affecting end organ function. Currently, most institutions will aim to avoid prolonged ECMO runs and will consider transition from ECMO to VAD within 7 to 10 days of support. A larger issue might be that the need for preimplantation ECMO reflects the overall severity of both end organ failure and myocardial failure.

In a recently reported multicenter study on VAD use in North America, BiVAD was used less frequently in the younger patients. ${ }^{1}$ This was consistent with our observed trend for infants $<1$ year old requiring BiVAD support less frequently, although this finding could have been confounded by a surgical bias and should be interpreted with caution.

Other factors associated with BiVAD use in our study included a non-DCM diagnosis and pre-existing RV failure, with a secondary impairment of hepatic and renal function. Combined together, these factors could be useful in highlighting high-risk patients who are more likely to develop severe RVD and need a BiVAD.

\section{BiVAD and Postoperative Morbidity and Mortality}

In both adult and pediatric reports in which BiVAD was used as rescue therapy for failing RV function, the BiVAD patients have had worse outcomes than those with an LVAD only. ${ }^{2,4}$ It is debatable whether the greater mortality with BiVAD use was because these were sicker patients with more organ compromise or whether having 2 devices resulted into more complications, irrespective of the preoperative status. Having 2 pumps, instead of 1 , increases the risk of infection and clot formation requiring more frequent pump changes. It also increases the postoperative bleeding, exposing patients waiting to be transplanted to high volume blood transfusions. ${ }^{2}$

Our study has shown that BiVAD patients are different to start with: they were sicker, often required ECMO preoperatively, and were more likely to have a diagnosis other than DCM. This would suggest that the greater mortality of children requiring BiVAD also reflected our current indications and highlights the importance of timely initiation of mechanical support. ${ }^{10,11}$

\section{Study Limitations}

Our results should be interpreted in the context of a retrospective study design. This affected our data collection; thus, some of the preoperative data collected retrospectively were not complete (eg, not all patients had undergone screening hepatic function tests before VAD implantation), which might have introduced bias. Second, the choice between LVAD and BiVAD was, in all but 1 case, determined by intraoperative RV performance, and 
we could not exclude a degree of selection bias in borderline patients with RVD.

\section{CONCLUSIONS}

The present study has demonstrated that RVD occurs in approximately $40 \%$ of pediatric VAD recipients and affects their postoperative morbidity and bridging outcomes. Children with RVD that was medically managed on LVAD had a complicated had a complicated postimplantation course; however, their overall survival was excellent. Severe RVD requiring BiVAD occurred in $25 \%$ patients, and those children had significantly reduced survival. Patients with non-DCM and those with impaired RV function and organ congestion preoperatively were more likely to develop RVD after VAD implantation. Preoperative ECMO and an elevated urea level were independent risk factors for BiVAD.

Additional research into the mechanisms and predictors of RVD after VAD implantation in children is needed and will help to optimize their perioperative management and the decision making between LVAD and BiVAD.

\section{References}

1. Fraser CD Jr, Jaquiss RD, Rosenthal DN, Humpl T, Canter CE, Blackstone EH, et al. Prospective trial of a pediatric ventricular assist device. N Engl J Med.2012; 367:532-41.

2. Morales DL, Almond CS, Jaquiss RD, Rosenthal DN, Naftel DC, Massicotte MP, et al. Bridging children of all sizes to cardiac transplantation: the initial multicenter North American experience with the Berlin Heart EXCOR ventricular assist device. J Heart Lung Transplant. 2011;30:1-8.

3. Hetzer R, Potapov EV, Stiller B, Weng Y, Hubler M, Lemmer J, et al, Improvement in survival after mechanical circulatory support with pneumatic pulsatile ventricular assist devices in pediatric patients. Ann Thorac Surg. 2006;82:917-24.

4. Morgan JA, John R, Lee BJ, Oz MC, Naka Y. Is severe right ventricular failure in left ventricular assist device recipients a risk factor for unsuccessful bridging to transplant and post-transplant mortality. Ann Thorac Surg. 2004;77:859-63.

5. Dang NC, Topkara VK, Mercando M, Kay J, Kruger KH, Aboodi MS, et al. Right heart failure after left ventricular assist device implantation in patients with chronic congestive heart failure. J Heart Lung Transplant. 2006;25:1-6.

6. Ochiai Y, McCarthy PM, Smedira NG, Banbury MK, Navia JL, Feng J, et al. Predictors of severe right ventricular failure after implantable left ventricular assist device insertion: analysis of 245 patients. Circulation. 2002;106:I-198-202.

7. Drakos SG, Janicki L, Horne BD, Kfoury AG, Reid BB, Clayson S, et al. Risk factors predictive of right ventricular failure after left ventricular assist device implantation. Am J Cardiol. 2010;105:1030-5.

8. Schenk S, McCarthy PM, Blackstone EH, Feng J, Starling RC, Navia JL, et al. Duration of inotropic support after left ventricular assist device implantation: risk factors and impact on outcome. J Thorac Cardiovasc Surg. 2006;131: 447-54.
9. Stiller B, Lemmer J, Schubery S, Ewert P, Schulze-Neick I, Hubler M, et al. Management of pediatric patients after implantation of the Berlin Heart EXCOR ventricular assist device. ASAIO J. 2006;52:495-500.

10. Almond CS, Morales DL, Blackstone EH, Turrentine MW, Imamura M, Massicotte MP, et al. Berlin Heart EXCOR pediatric ventricular assist device for bridge to heart transplantation in US children. Circulation. 2013;127: 1702-11.

11. Holman WL, Kormos RL, Naftel DC, Miller MA, Pagani FD, Blume E, et al. Predictors of death and transplant in patients with a mechanical circulatory suppor device: a multi-institutional study. J Heart Lung Transplant. 2009;28:44-50.

12. Matthews JC, Koelling TM, Pagani FD, Aaronson KD. The right ventricular failure risk score: a pre-operative tool for assessing the risk of right ventricular failure in left ventricular assist device candidates. J Am Coll Cardiol. 2008;51: 2163-72.

13. Aissaoui N, Morshuis M, Schoenbrodt M, Hakim Meibodi K, Kizner L, Börgermann J, et al. Temporary right ventricular mechanical circulatory support for the management of right ventricular failure in critically ill patients. $J$ Thorac Cardiovasc Surg. 2013;146:186-91.

14. Leeuwenburgh BP, Helbing WA, Steendijk P, Schoof PH, Baan J. Effects of acute left ventricular unloading on right ventricular function in normal and chronic right ventricular pressure-overloaded lambs. J Thorac Cardiovasc Surg. 2003; $125: 481-90$

15. Kormos RL, Teuteberg JJ, Pagani FD, Russell SD, John R, Miller LW, et al Right ventricular failure in patients with the HeartMate II continuous-flow left ventricular assist device: incidence, risk factors, and effect on outcomes. J Thorac Cardiovasc Surg. 2010;139:1316-24.

16. Stein ML, Robbins R, Sabati AA, Reinhartz O, Chin C, Liu E, et al. Interagency Registry for Mechanically Assisted Circulatory Support (INTERMACS)-defined morbidity and mortality associated with pediatric ventricular assist device support at a single US center: the Stanford experience. Circ Heart Fail. 2010; 3:682-8.

17. Potapov EV, Stepanenko A, Dandel M, Kukucka M, Lehmkuhl HB, Weng Y, et al. Tricuspid incompetence and geometry of the right ventricle as predictors of right ventricular function after implantation of a left ventricular assist device. J Heart Lung Transplant. 2008;27:1275-81.

18. Puwanant S, Hamilton KK, Klodell CT, Hill JA, Schofield RS, Cleeton TS, et al. Tricuspid annular motion as a predictor of severe right ventricular failure after left ventricular assist device implantation. J Heart Lung Transplant. 2008;27: 1102-7.

19. Fan Y, Zhang AM, Weng YG, Huebler M, Miera O, Franz N, et al. Factors associated with the need of biventricular mechanical circulatory support in children with advanced heart failure. Eur J Cardiothorac Surg. 2013;43:1028-35.

20. Romano MA, Cowger J, Aaronson KD, Pagani FD. Diagnosis and managemen of right-sided heart failure in subjects supported with left ventricular assist devices. Curr Treat Options Cardiovasc Med. 2010;12:420-30.

21. Van Meter CH Jr. Right heart failure: best treated by avoidance. Ann Thorac Surg. 2001:71:220-2.

22. Potapov E, Meyer D, Swaminathan M, Ramsay M, El Banayosy A, Diehl C, et al Inhaled nitric oxide after left ventricular assist device implantation: a prospective, randomized, double-blind, multicenter, placebo-controlled trial. J Heart Lung Transplant. 2011;30:870-8.

23. Gandhi SK, Huddleston CB, Balzer DT, Epstein DJ, Boschert TA, Canter CE. Biventricular assist devices as a bridge to heart transplantation in small children. Circulation. 2008;118:S89-93.

24. Chen JM, Richmond ME, Charette K, Takayama H, Williams M, Gilmore L, et al. A decade of pediatric mechanical circulatory support before and after cardiac transplantation. J Thorac Cardiovasc Surg. 2012;143:344-51. 
TABLE E1. Echocardiographic characteristics of patients with LVAD with and without RVD and BiVAD

Echocardiographic

$$
\text { variables }
$$

Right atrial area (Z-score)

Left atrial area (Z-score)

TR grade

$\operatorname{TR} \operatorname{Vmax}(\mathrm{m} / \mathrm{s})$

TV annulus (Z-score)

RV function

RVEDD (Z-score)

LVEDD (Z-score)
LVAD only

0.54 (-3.27 to 7.86$)$

3.55 ( -1.17 to 15.62$)$

1 (0 to 3$)$

2.8 (2.2 to 4.0$)$

$1.24(-3.98$ to 26$)$

0 (0 to 2$)$

1.67 ( -5.8 to 6.02$)$

\section{LVAD-RVD}

$1.55(-5.52$ to 7.82$)$

$9.72(0.05$ to 18.55$)$

2 (0 to 4$)$

2.7 (1.9 to 4.4$)$

0.12 ( -3.05 to 12.29$)$

0.5 (0 to 3$)$

2.54 (1.48 to 12.35$)$

$5.88(-4.48$ to 11.37$)$
BIVAD

$P$ value

$1.2(-0.95$ to 22.37$)$

$2.30(-1.86$ to 13.02$)$

1.5 (0 to 4$)$

2.81 (1.8 to 3.0$)$

2.45 ( -2.59 to 9.77$)$

2 (0 to 3$)$

3.01 ( -1.1 to 7.82$)$

$5.90(-3.76$ to 12.65$)$

.38

.38
.46

\section{.46}

.32

.79

.71

.01

.12

.81

Data presented as median (range). Significance testing among the 3 groups was performed using the Kruskal-Wallis test. Ventricular function was qualitatively graded as follows: 0 , normal; 1, mild; 2, moderate; and 3, severe. $L V A D$, Left ventricular assist device; RVD, right ventricular dysfunction; BiVAD, biventricular assist device; TR, tricuspid regurgitation (graded as follows: 0 , none; 1 , trivial; 2 , mild; 3 , moderate; and 4, severe); Vmax, maximal velocity; $T V$, tricuspid valve; $R V$, right ventricular; $L V$, left ventricular; $E D D$, end-diastolic dimension. 